\title{
Primary pelvic hydatid cyst presenting with obstructive uropathy and renal failure
}

\author{
V. Seenu, M.C. Misra, S.C. Tiwari ${ }^{2}$, R. Jain ${ }^{1}$ and C. Chandrashekhar \\ Departments of Surgical Disciplines, ${ }^{1}$ Radiodiagnosis and ${ }^{2}$ Nephrology, All India Institute of Medical \\ Sciences, Ansari Nagar, New Delhi, India
}

\begin{abstract}
Summary: Primary pelvic hydatid cyst is a rare entity. Pelvic hydatid cysts usually present with pressure symptoms involving adjacent organs (bladder and rectum usually). A case of primary pelvic hydatid cyst presenting with obstructive uropathy leading to chronic renal failure is presented. A combination of preoperative albendazole therapy of $1.2 \mathrm{~g} /$ day for 8-12 weeks and surgical excision were effective in alleviating the symptoms and improving the renal function.
\end{abstract}

\section{Introduction}

Primary pelvic hydatid cysts are rare. Bickers ${ }^{1}$ reported the occurrence of pelvic hydatid cyst in 12 out of 532 cases of proven hydatid disease $(2.25 \%)$. Clements $^{2}$ reported two cases of primary pelvic hydatid cysts in a series of 43 patients with pelvic hydatid cysts. Pelvic hydatid cysts usually present with pressure effects on adjacent organs such as the bladder and rectum. Obstructive uropathy and renal failure is an uncommon presentation. Morris $^{3}$ described an intra-abdominal cyst causing renal failure in a 10 year old child. We present a case of primary pelvic hydatid cyst with obstructive uropathy and renal failure.

\section{Case report}

A 38 year old male presented with difficulty in passing urine for the last 15 years and generalized oedema for one month. There was a history of oliguria, sensation of incomplete emptying and terminal dribbling of urine. The patient had a good urinary stream but he had to apply pressure over the suprapubic region to empty the bladder completely. He also had anorexia for 2 months but no history of fever with chills, haematuria or pyuria. He was operated on in a military hospital 11 years previously for the same problem but details of the surgery were not known.

On examination, he had pallor, facial puffiness and anasarca. Tachypnoea was present with a respiratory rate of 30 per minute, but there was no peripheral or central cyanosis. Pulse rate (90/

Correspondence: M.C. Misra, M.S.

Accepted: 28 April 1994 minute) and blood pressure $(130 / 80 \mathrm{mmHg})$ were normal. Abdominal examination revealed a smooth, firm, non-tender and non-mobile suprapubic mass reaching up to the umbilicus. On per rectal examination, a smooth spherical mass was felt anteriorly in the rectovesical pouch.

Investigations revealed haemoglobin $6.7 \mathrm{~g} / \mathrm{dl}$ total leucocyte count $5.4 \times 10^{9} / 1$, blood urea $35 \mathrm{mmol} / 1$, serum creatinine $1,167 \mu \mathrm{mol} / 1$, and serum sodium and potassium 138 and $4.2 \mathrm{mmol} / \mathrm{l}$, respectively. Urine examination showed pus cells (4-5/HPF) but the urine culture was sterile. Chest $\mathrm{X}$-ray was normal. Ultrasound examination revealed a cystic mass in the pelvis suggestive of a hydatid cyst with bilateral hydroureteronephrosis. Liver and spleen were normal. Computerized tomographic (CT) scan revealed a pelvic hydatid cyst with bilateral hydroureteronephrosis (Figures 1 and 2). Indirect haemagglutination antigen (IHA) test for hydatid disease was positive. Cystopanendoscopy showed extrinsic compression in the bladder neck region. Trigone and ureteric openings could not be examined due to distortion by the extrinsic pressure.

Bilateral percutaneous nephrostomy was done to relieve the urinary tract obstruction. Simultaneously, the patient was put on albendazole $(15 \mathrm{mg} / \mathrm{kg} /$ day $)$ for 6 weeks. Reassessment by ultrasound after 6 weeks showed decompression of the pelvi-calyceal system and renal biochemical parameters showed gradual improvement. CT scan revealed reduction in the size of the cyst with appreciable increase in the density of the contents as well as a reduction in the number and size of the daughter cysts (Figure 3). Right and left percutaneous nephrostomy tubes were dislodged accidentally 2 and 6 months after insertion, respec- 


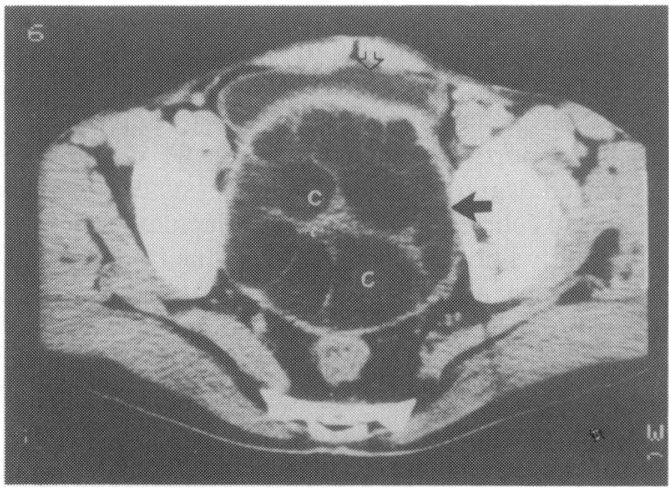

Figure 1 CT scan of pelvis showing a large hydatid cyst (solid arrow) with multiple daughter cysts (c) compressing and displacing the urinary bladder (open arrow) anteriorly.

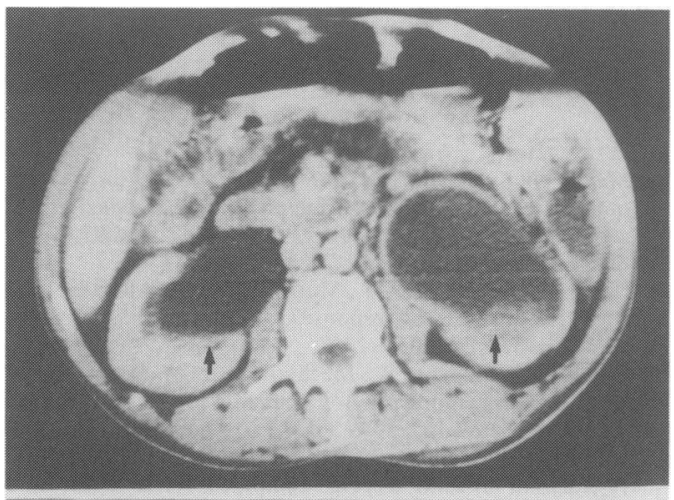

Figure 2 CT scan at the level of the kidneys showing bilateral gross hydronephrosis with delay in contrast excretion (solid arrows).

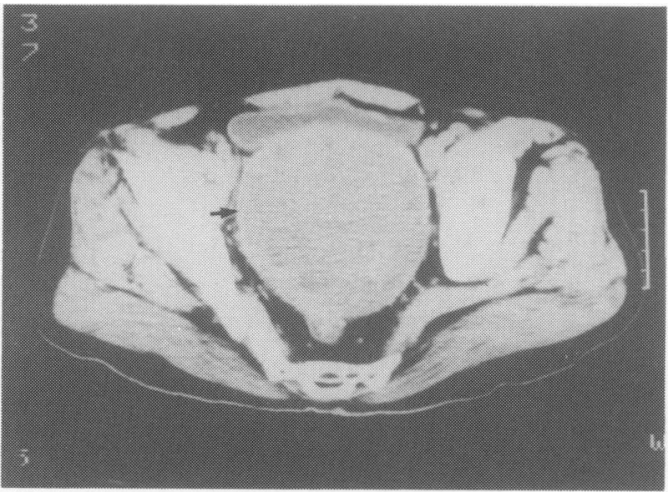

Figure 3 CT scan of the pelvis 6 weeks after albendazole therapy. The cyst (solid arrow) is marginally reduced in size. The overall density of the cyst contents has increased dramatically. The number and size of the daughter cysts has reduced significantly. tively. On subsequent follow-up, there was no increase in hydronephrosis and renal function continued to improve (serum creatinine fell to $454 \mu \mathrm{mol} / \mathrm{l})$. Six months after albendazole therapy, the mass became impalpable per abdomen but per rectal examination findings remained unchanged.

Despite radiological and clinical improvement, the patient had no improvement in the subjective urinary symptoms. Repeat investigations done at this stage revealed haemoglobin $9.2 \mathrm{~g} / \mathrm{dl}$, blood urea $14.6 \mathrm{mmol} / \mathrm{l}$ and serum creatinine $454 \mu \mathrm{mol} / \mathrm{l}$. Exploratory laparotomy was done after haemodialysis. At surgery, a cyst was found in the rectovesical pouch, which had dense adhesions with the urinary bladder anteriorly and rectum posteriorly. Cyst contents, which were putty like, were evacuated after packing of the peritoneal cavity and the cyst was washed with povidone iodine. Omentoplasty was performed to obliterate the residual cyst. Postoperative recovery was uneventful and the patient was discharged on the eighth postoperative day. Albendazole ( $1.2 \mathrm{~g} /$ day) was continued for 1 month in the postoperative period. A CT scan performed 6 months after surgery revealed complete resolution of hydroureteronephrosis with normal renal cortical thickness and contrast excretion. The urinary bladder regained its normal capacity and distensibility. Serum creatinine, which was $381 \mu \mathrm{mol} / 1$ at the time of discharge from hospital came down to $166 \mu \mathrm{mol} /$ 1 at 18 months follow-up. The patient was also free of his earlier distressing urinary symptoms.

\section{Discussion}

Intraperitoneal hydatid cysts are usually secondary to the rupture (spontaneous or accidental at surgery) of a primary hydatid cyst (in the liver usually). A solitary cyst in the pelvic cavity can be considered primary only when no other cysts are present in the common sites of occurrence. In such a case, the hydatid embryo gains access to the pelvis by haematogenous or lymphatic route. ${ }^{4}$ In our patient, there was a hydatid cyst in the pelvis and there were no other hydatid cysts (in the liver, spleen, kidney, lungs or peritoneal cavity). It can thus be considered as a primary pelvic hydatid cyst.

Pelvic hydatid cysts usually present as a nonspecific mass with pressure effects on adjacent organs such as the rectum and urinary bladder. ${ }^{5}$ Rarely, they can cause obstructed labour, ${ }^{1}$ obstructive uropathy ${ }^{6}$ and renal failure. ${ }^{3}$ Sometimes, they can rupture spontaneously. ${ }^{7}$ Hydatid disease should be considered in the differential diagnosis of unusual cystic swellings in the pelvis, particularly in a patient who has lived in an endemic area. Serological tests help in confirming the diagnosis. US and CT scan may help to determine the exact 
location and nature of a cystic pelvic mass, and also exclude hydatid disease elsewhere.

Drug treatment using albendazole has been found to be successful in a proportion of cases but drug therapy is generally not used as the primary treatment except in cases where the patient is not fit for surgery. Combination of preoperative albendazole therapy, surgery and postoperative albendazole therapy (for 6-8 weeks, to avoid recurrence of the cyst) is a useful regime. Morris et al. ${ }^{8}$ showed that albendazole suppresses the development of hydatid cysts following intraperitoneal inoculation of protoscolices in experimental model.

Morphological changes in hydatid cysts following mebendazole or albendazole therapy have been studied by some authors. ${ }^{9-11}$ Complete disappearance of cysts occurs in many cases and is the most reliable sign of response. With partial response, reduction in size of the cyst, decrease in cyst tension, increased echogenicity of the cyst contents and thickening or calcification of the cyst wall can occur. Todorov et al. ${ }^{10}$ have noticed increased echogenicity on ultrasound as well as increased density on CT scan (from near watery to about 40-50 Hounsfield units) of the cyst contents in a small proportion of their cases. The reason for decreased water content of the cysts leading to increased echogenicity as well as decrease in size is not exactly known but it could be due to decreased secretion (damage to the germinal membrane), resorption or leakage of fluid from the cysts (due to disruption of the germinal membrane). Operative appearance of the cyst contents in such cases has not been mentioned. It is thus difficult to comment about the exact changes occurring in the cyst contents leading to increased echogenicity. In our case, there was prominent increase in echogenicity with a reduction in cyst size. The contents had become putty like but there was no evidence of bleeding. Whether infection had a role to play in causing these changes cannot be ruled out but no frank pus was seen.

\section{References}

1. Bickers, W.M. Hydatid disease of the female pelvis. Am J Obstet Gynecol 1970, 107: 477-483.

2. Clements, R. \& Bowyer, F.M. Hydatid disease of pelvis. Radiology 1986, 37: 375-377.

3. Morris, D.G. Intraabdominal hydatid cyst causing renal failure. Br J Surg 1952, 140: 42.

4. Salvaggi, F.P., Fabiano, G., Santa Croce, S. \& Tragicante, A A retrovesical echinococcal cyst. Unusual cause of urinary retention. Eur J Urol 1978, 4: 60-62.

5. Ptasznik, R. \& Henessey, O.F. Pelvic hydatid disease presenting as acute urinary obstruction. $\mathrm{Br} J$ Radiol 1988, 61: 164-165.

6. Birkoff, J.D. \& McClean, B.L. Echinococcal disease of the pelvis: urologic complication, diagnosis and treatment. $J$ Urol 1973, 109: 473-476.

7. Vaidyanathan, S., Rao, M.S., Sharma, S.K. et al. Nonoperative management of pelvic hydatid cyst communicating with bladder. $J$ Urol 1979, 121: 245-247.

8. Morris, D.L., Chinnery, J.B., Hardcastle, J.D. et al. Can albendazole reduce the risk of implantation of spilled protoscolices? An animal study. Trans $R$ Soc Trop Med Hyg 1986, 80: 481-484.

9. Morris, D.L., Dykes, P.W., Marriner, S. et al. Albendazole objective evidence of response in human hydatid disease. JAMA 1985, 253: 2053-2057.

10. Todorov, T., Vutova, K., Mechkov, G. et al. Evaluation of response to chemotherapy of human cystic echinococcosis. Br J Radiol 1990, 63: 523-531.

11. Morris, D.L., Skene-Smith, H., Haynes, A. \& Burrows, F.G. Abdominal hydatid disease: computed tomographic and ultrasound changes during albendazole therapy. Clin Radiol 1984, 35: 297-300. 